\title{
Broad and comprehensive approach to evaluate infrared intensities at atomic level: the $\mathrm{AC} / \mathrm{DC}$ analysis
}

\author{
Wagner E. Richter ${ }^{a, b}$, Leonardo J. Duarte ${ }^{b}$, Luciano N. Vidal $^{c}$, Roy E. Bruns ${ }^{b}$ \\ $a$ - Department of Chemical Engineering, Federal University of Technology - Paraná. Ponta Grossa-PR, Brazil. \\ $b$ - Institute of Chemistry, State University of Campinas. Campinas-SP, Brazil. \\ $c$ - Department of Chemistry, Federal University of Technology - Paraná. Curitiba-PR, Brazil.
}

\begin{abstract}
We present a complete theoretical protocol to split infrared intensity in terms owing to individual atoms in two different but related approaches: the Atomic Contributions (AC's) show how the entire molecule motion is noticed by the electronic structure of a single atom, and therefore reflected on the intensity. On the other hand, the Dynamic Contributions (DC's) show how the displacement of a single atom is noticed by the electronic structure of the entire molecule, and reflected on the IR intensity. The two analyses are complementary ways of partitioning the same total intensity, and conserve most of the features of the total intensity itself. Combined they are called the $A C / D C$ analysis. These can be further partitioned following the CCTDP (or CCT) models regarding the population analysis chosen by the researcher. The main conceptual features of the equations are highlighted and representative numerical results are shown to support the interpretation of the equations. The results are invariant to rotation and translation and can readily be extended to molecules of any size, shape or symmetry. A fully automated protocol managed by Placzek program is made available, free of charge.
\end{abstract}

\section{Corresponding author}

Prof. Dr. Wagner Eduardo Richter

Department of Chemical Engineering

Federal University of Technology - Paraná

e-mail: richter@utfpr.edu.br 


\section{Introduction}

Infrared (IR) Spectroscopy is a major technique to evaluate chemical atomic and electronic structures. The vibrational features of a molecule depend not only of the positions of nuclei in space but also, and expressively, on electronic properties like total charge, polarizability and distribution of the electronic density around the molecule. Therefore, comparisons between theoretical and experimental vibrational assignments are widely used to infer about the reliability of theoretical approaches. Particularly, the relation between vibrational frequencies and the bond strength of a characteristic chemical group is now deeply stablished. Interestingly, the normal coordinates usually invoked to describe the vibrations show all atoms being displaced from their equilibrium positions, even though we associate a given experimental frequency to a particular chemical group within the molecule whose individual vibration is its main descriptor.

Following this line, IR intensities (also known as band strengths) are related to electronic properties of the vibrating molecular system. Thus, one could argue whether the IR intensities of a given vibrational mode could be assigned to specific atoms or chemical groups. We already proposed a methodology in which IR intensities may be splitted into atomic, non-overlapping terms, each of them measured in the same unit $\left(\mathrm{km}^{\mathrm{mol}} \mathrm{mol}^{-1}\right)$ as the total intensity [1]. The purpose of the present work is to generalize those conclusions in a wider and deeper way, showing that are not only one, but two different, although complementary, ways of performing it. Moreover, the information each of these procedures is different, as well as the assessment to them through theoretical methods.

\section{Theory}

\subsection{Derivation of atomic and dynamic contributions}

We begin with no more than the classical treatment given at Molecular Vibrations by Wilson, Decius \& Cross $[2,3]$. For instance, consider an arbitrary $N$-atom molecule and its $3 N-6$ different vibrational modes $(3 N-5$ if it's linear); within the double harmonic approximation, the infrared intensity of the $k^{\text {th }}$ vibrational mode is given by:

$$
A_{k}=\left(\frac{N_{A} \pi}{3 c^{2}}\right)\left(\frac{\partial \vec{p}}{\partial Q_{k}}\right)^{2}
$$

for which $N_{A}$ is the Avogadro's constant, $c$ is the speed of light, $\vec{p}$ is the molecular electric dipole moment vector and $Q_{k}$ is the $k^{t h}$ normal coordinate associated to that given vibrational mode.

Our main interest here concerns the assessment of the IR intensities at atomic level. In order to pursue that, the dipole moment derivatives that appear in Eq. (1) are usually computed in a two-step calculation: this derivative is firstly evaluated with respect to individual displacements of all atoms in the Cartesian space and then transformed to the normal coordinate basis. Mathematically:

$$
\left(\frac{\partial \vec{p}}{\partial Q_{k}}\right)=\sum_{j=1}^{N}\left(\frac{\partial \vec{p}}{\partial \vec{r}_{j}}\right) \cdot\left(\frac{\partial \vec{r}_{j}}{\partial Q_{k}}\right)=\sum_{j=1}^{N}\left(\frac{\partial \vec{p}}{\partial Q_{k}}\right)_{(j)}
$$

in which $\frac{\partial \vec{p}}{\partial \vec{r}_{j}}$ stands for the dipole moment derivative which respect to Cartesian displacements of the $j^{\text {th }}$ atoms, while $\frac{\partial \vec{r}_{j}}{\partial Q_{k}}$ transforms these Cartesian displacements on their correct amplitudes within the normal coordinate. We have adopted this symbology to keep the equations as concise as possible. Thus, the terms appearing in the 
latter equations must be interpreted as:

$$
\begin{aligned}
&\left(\frac{\partial \vec{p}}{\partial \vec{r}_{j}}\right)= {\left[\begin{array}{lll}
\left(\frac{\partial p_{x}}{\partial x_{j}}\right) & \left(\frac{\partial p_{x}}{\partial y_{j}}\right) & \left(\frac{\partial p_{x}}{\partial z_{j}}\right) \\
\left(\frac{\partial p_{y}}{\partial x_{j}}\right) & \left(\frac{\partial p_{y}}{\partial y_{j}}\right) & \left(\frac{\partial p_{y}}{\partial z_{j}}\right) \\
\left(\frac{\partial p_{z}}{\partial x_{j}}\right) & \left(\frac{\partial p_{z}}{\partial y_{j}}\right) & \left(\frac{\partial p_{z}}{\partial z_{j}}\right)
\end{array}\right] } \\
&\left(\frac{\partial \vec{r}_{j}}{\partial Q_{k}}\right)=\left[\begin{array}{l}
\left(\frac{\partial x_{j}}{\partial Q_{k}}\right) \\
\left(\frac{\partial y_{j}}{\partial Q_{k}}\right) \\
\left(\frac{\partial z_{j}}{\partial Q_{k}}\right)
\end{array}\right]
\end{aligned}
$$

Our group already presented a result absolutely equivalent to Eq. (2) earlier, in 2015 [1]; at that time, we called it Dynamic Atomic Contribution of the $j^{\text {th }}$ atom to the (total) dipole moment derivative. For reasons that will became clearer as the reader progresses on this text, we'll now shorten it to only Dynamic Contribution, DC. A brief explanation would consider that this DC term reflects the dynamics of the $j^{\text {th }}$ atom as reflected in the entire molecular dipole moment derivative. Such way of partitioning the total dipole moment derivative in atomic terms therefore arises from the individual atomic motions along the normal coordinate, which embrace the whole molecule.

An alternative way of expressing the total dipole moment derivative as a sum of single-atom contributions is based on the atomic partitioning of the molecular dipole moment instead of the normal coordinate. Each term would be different, but the overall sum needs to be necessarily equivalent, since they reflect the same property. However, while the molecular dipole and the molecular electronic density are well defined entities, the atomic partitioning of them can be carried out in several different ways. Each of them gives rise to a different population analysis which, after being combined to the nuclei's charges, are then interpreted as partial atomic charges. Theoretical and computational chemists readily recognize the most widely used: Mulliken [4], MBS [5], MK [6], NPA [7], Chelp/ChelpG [8, 9], DMA [10], GAPT [11], Voronoi [12], CM5 [13], Hirshfeld/Hirshfeld-I $[14,15]$ and QTAIM [16], to name just a few. New models continue to appear in the literature and are rapidly implemented in all sorts of computational codes.

Regardless the charge model, all of them can be placed into one of two main groups:

1. Only-charge models, which reproduce the molecular dipole moment only by means of individual charges on the atoms, usually centered at the nuclei's positions;

2. Charge-and-dipole models, which reproduce the dipole moment using not only point charges, but also intra-atomic dipole moments accounting for the eventual asymmetry of the electronic distribution within the atom.

Mathematically, one has:

$$
\vec{p}=\sum_{i=1}^{N} \vec{p}_{i} \quad \begin{cases}\vec{p}=\sum_{i=1}^{N}\left(q_{i} \cdot \vec{r}_{i}\right) & \text { (charge-only models) } \\ \vec{p}=\sum_{i=1}^{N}\left(q_{i} \cdot \vec{r}_{i}+\vec{m}_{i}\right) & \text { (charge-and-dipole models) }\end{cases}
$$

The immediate consequence of Eq. (5) is:

$$
\left(\frac{\partial \vec{p}}{\partial Q_{k}}\right)=\sum_{i=1}^{N}\left(\frac{\partial \vec{p}_{i}}{\partial Q_{k}}\right)
$$

Eq. (6) is what now we call Atomic Contribution (AC) of the $i^{\text {th }}$ atom to the molecular dipole moment derivative. Oppositely to the Dynamic Contributions, the Atomic Contributions express the effect of the entire 
molecular vibration within a single atom's electronic density. These acronyms started as a coincidence (both emerge from the earlier name Dynamic Atomic Contribution, DAC), but in view of the $40^{\text {th }}$ anniversary of Back in Black album, firstly released in 1980, we considered it would be nice to call it the $A C / D C$ analysis. Both are different, though complementary, ways of evaluating IR intensities at the atomic level:

1. Atomic Contribution, $\mathrm{AC}$ :

(a) The effect, on the electronic structure of the $i^{\text {th }}$ atom, caused by the displacements of all atoms, or

(b) The effect, on the electronic structure of the $i^{\text {th }}$ atom, caused by the vibration of the entire molecule.

(c) AC's split the numerator of $\frac{\partial \vec{p}}{\partial Q_{k}}$ into $N$ terms, while the denominator concerns the entire molecular motion;

(d) AC's show which atomic electron densities are the more and the less affected by the molecular vibration, in the passive sense.

2. Dynamic Contribution, DC:

(a) The effect, on the combined electronic densities of all atoms, caused by the displacements of only one (the $j^{\text {th }}$ ) atom, or

(b) The effect, on the entire molecule, of the vibration of only one (the $j^{\text {th }}$ ) atom.

(c) DC's split the denominator of $\frac{\partial \vec{p}}{\partial Q_{k}}$ into $N$ terms, while the numerator concerns the entire molecular dipole moment;

(d) DC's show which atoms affect more or less the whole molecular dipole while moving, in the active sense.

Notice that whenever we want to deal with AC's, the index $i$ is used, and whenever we want to deal with DC's, the $j$ index is used instead. To help the reader follow our equations let us take advantage of visual and graphical approaches. For simplicity, let us consider a planar molecule (like ethene, $C_{2} H_{4}$ ), placed within the $x y$ plane, and look one of the out-of-plane vibrations. The total dipole moment derivative, in this case, is resumed to its only non-null component along the $z$-axis, caused by the displacements of the atoms also solely along this direction; Figure 1 shows these differences with colors.
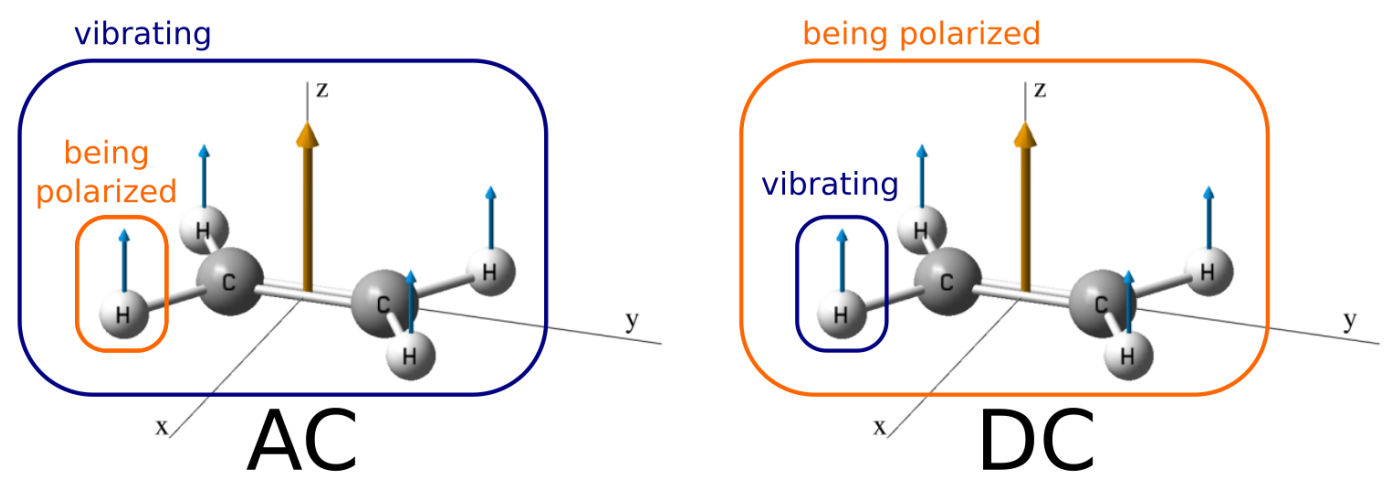

Figure 1: LEFT. Atomic Contributions (AC) measure the polarization change on a single atom when the entire molecule vibrates along the normal coordinate. RIGHT. Dynamic Contributions (DC) measure of the polarization change on the entire molecule when a single atom is displaced along the normal coordinate. The large orange arrow is the total dipole moment derivative for the out-of-plane bending of ethene, while the small blue arrows are the directions of the displacement for the hydrogen atoms.

As the aforementioned equations show, this $z$-component of the total dipole moment derivative can be 
expanded in two ways:

$$
\begin{aligned}
\left(\frac{\partial p_{z}}{\partial Q_{k}}\right) & =\sum_{i=1}^{N}\left(\frac{\partial p_{z, i}}{\partial Q_{k}}\right) & & \text { (the AC's) } \\
& =\sum_{j=1}^{N}\left(\frac{\partial p_{z}}{\partial z_{j}}\right)\left(\frac{\partial z_{j}}{\partial Q_{k}}\right) & & \text { (the DC's) }
\end{aligned}
$$

for which the sum over $i$ stands for the $i^{\text {th }}$ atomic contribution to the molecular dipole moment (the electronic part), while the sum over $j$ stands for the displacement of the $j^{\text {th }}$ atom along the $k^{\text {th }}$ normal coordinate (the mechanical part). Combining the two equations results in:

$$
\begin{aligned}
\left(\frac{\partial p_{z}}{\partial Q_{k}}\right) & =\sum_{i=1}^{N} \sum_{j=1}^{N}\left(\frac{\partial p_{z, i}}{\partial z_{j}}\right)\left(\frac{\partial z_{j}}{\partial Q_{k}}\right) \\
& =\sum_{j=1}^{N} \sum_{i=1}^{N}\left(\frac{\partial p_{z, i}}{\partial z_{j}}\right)\left(\frac{\partial z_{j}}{\partial Q_{k}}\right)
\end{aligned}
$$

Eqs. (9) and (10) are totally equivalent in the mathematical sense but were written with their sums interchanged. Therefore, the results obtanied through them need to be necessarily equivalent, but the interpretations of the inner terms do not. For instance, let us consider an arbitrary term of the first sum in Eq. (9); it will contains all $z_{j}$ terms, from $j=1$ up to $j=N$, within a single $i$ : it is $i^{\text {th }}$ atomic contribution, $\operatorname{AC}(i)$. On the other hand, an arbitrary term from the first sum in Eq. (10) contains all the $p_{i}$ terms, also from $i=1$ up to $i=N$, for that given $j$ : this is the $j^{\text {th }}$ dynamic contribution, $\operatorname{DC}(j)$. If $i$ and $j$ are expanded perpendicularly, it is possible to distribute Eqs. (9) and (10) as a squared array, with each of them being a sum over rows or columns, with subsequent multiplication by the appropriate(s) $\frac{\partial z_{j}}{\partial Q_{k}}$ terms:

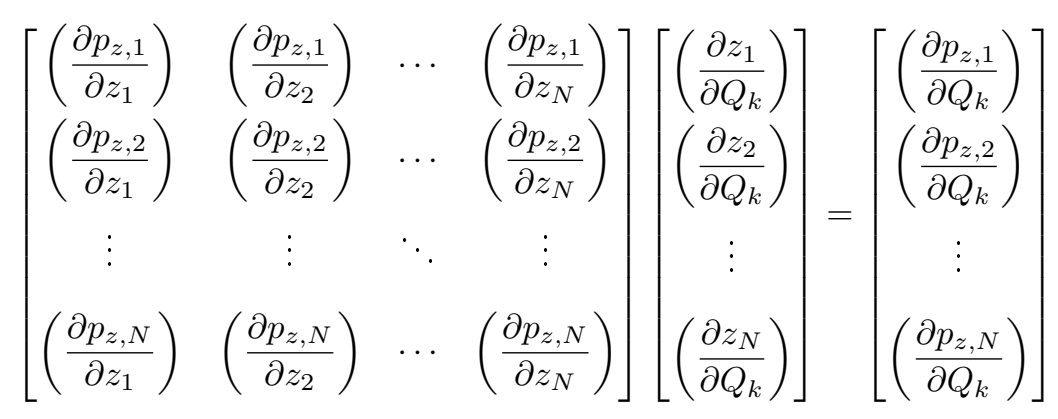

Thus, the AC's and DC's will be obtained by the terms given in Figure 2 multiplied by the respective $\frac{\partial z_{j}}{\partial Q_{k}}$ elements and then summing:

\begin{tabular}{|cccc|c|}
\hline$\left[\left(\frac{\partial p_{z, 1}}{\partial z_{1}}\right)\right.$ & $\left(\frac{\partial p_{z, 1}}{\partial z_{2}}\right)$ & $\cdots$ & $\left(\frac{\partial p_{z, 1}}{\partial z_{N}}\right)$ & $\mathrm{AC}(1)$ \\
\hline$\left(\frac{\partial p_{z, 2}}{\partial z_{1}}\right)$ & $\left(\frac{\partial p_{z, 2}}{\partial z_{2}}\right)$ & $\cdots$ & $\left(\frac{\partial p_{z, 2}}{\partial z_{N}}\right)$ & $\mathrm{AC}(2)$ \\
\hline$\vdots$ & $\vdots$ & $\ddots$ & $\vdots$ & \\
\hline$\left(\frac{\partial p_{z, N}}{\partial z_{1}}\right)$ & $\left(\frac{\partial p_{z, N}}{\partial z_{2}}\right)$ & $\cdots$ & $\left(\frac{\partial p_{z, N}}{\partial z_{N}}\right)$ & $\mathrm{AC}(\mathrm{N})$ \\
\hline
\end{tabular}

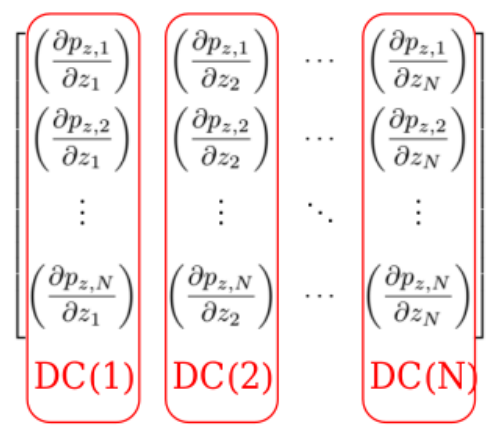

Figure 2: How the Atomic and Dynamic contributions can be viewed in a square array expanded both ways over the $N$ atoms in the molecule. AC's are the sum of the effect of the entire molecular vibration on a single atom; DC's is the effect on the entire molecule caused by the displacement of a single atom. 
The careful reader will notice that the direct substitution of Eqs. (2) and (6) into Eq. (1) would lead to cross-terms due to the quadratic dependence on the total dipole moment derivative. We can avoid that by substituting just one of these derivatives, so:

$$
\begin{aligned}
A_{k} & =\left(\frac{N_{A} \pi}{3 c^{2}}\right)\left(\frac{\partial \vec{p}}{\partial Q_{k}}\right)^{2} \\
& =\left(\frac{N_{A} \pi}{3 c^{2}}\right)\left(\frac{\partial \vec{p}}{\partial Q_{k}}\right) \cdot\left(\frac{\partial \vec{p}}{\partial Q_{k}}\right) \\
& =\left(\frac{N_{A} \pi}{3 c^{2}}\right) \sum_{i=1}^{N}\left(\frac{\partial \vec{p}}{\partial Q_{k}}\right) \cdot\left(\frac{\partial \vec{p}_{i}}{\partial Q_{k}}\right)=\sum_{i=1}^{N} A_{i, k} \quad \text { (the AC's) } \\
& =\left(\frac{N_{A} \pi}{3 c^{2}}\right) \sum_{j=1}^{N}\left(\frac{\partial \vec{p}}{\partial Q_{k}}\right) \cdot\left(\frac{\partial \vec{p}}{\partial Q_{k}}\right)_{(j)}=\sum_{j=1}^{N} A_{j, k} \quad \text { (the DC's) }
\end{aligned}
$$

By doing so, one can express the total IR intensity of the $k^{\text {th }}$ vibrational mode as a sum of contributions, each of them belonging to a single atom, without cross-terms and in the same units $\left(\mathrm{km}^{\mathrm{m}} \mathrm{mol}^{-1}\right)$ as the total intensity. If another unit is preferred over $\mathrm{km}_{\mathrm{mol}} \mathrm{mol}^{-1}$, it is just a matter of employing the correct transformation between them, with no harm to the conceptual interpretations.

It is important to notice that the total IR intensity of a given mode will be always positive (because of the squared dependence of Eq. (1)), but the individual AC's and DC's (in $\mathrm{km}_{\mathrm{mol}}^{-1}$ ) can be either positive or negative. A good manner to understand this is to visualize this squared derivative as actually two equal vectors coupling. The vectors themselves might be either positive or negative (the well known problem of the dipole moment derivative's signal), but in both cases the coupling will result in something positive. However, the individual (AC's or DC's) terms don't need to be necessarily of the same sign of the total derivative, thus the coupling may be either positive or negative. Regardless the sign, the sum of all AC's or DC's must agree with the total intensity of that given vibrational mode.

\subsection{Choosing the charge model: different AC's, same DC's}

Now a question emerges: how the differences among the several charge models one could choose within Eq. (5) would propagate inside the $\mathrm{AC} / \mathrm{DC}$ results? The short answer is:

There are multiple ways in which one can express the molecular dipole moment. Each of these (the charge models) will deliver different results for the Atomic Contributions (AC). Conversely, there is only a single way of expressing the molecular motion in the Cartesian space, thus only a given set of results for the DC's, regardless the chosen charge model.

The DC's will only be dependent of the charge model if this model do not reproduce the molecular dipole moment; this situation, although possible, most researchers by all means try to avoid. Therefore, if we perform calculations for the a given molecule under two different charge partition schemes, the AC's will reflect these differences, while the DC's won't. Examples to fully demonstrate this will be presented at next section.

Since the AC's and DC's came both from the total dipole moment derivative, and considering this total derivative can be divided into terms regarding the different charge models used, seems natural that the AC/DC results can be divided in the same way. Let us develop the general equations for the two situations exhibited in Eq. (5): charge-only and charge-and-dipole models. We will demonstrate the second case since the first one can be readily obtained from it by simple cancellation of the dipole-dependent terms. 


\subsubsection{Charge - and - dipole models}

Models employing both atomic charges and atomic dipoles express the total dipole as follows:

$$
\vec{p}=\sum_{i=1}^{N}\left(q_{i} \cdot \vec{r}_{i}+\vec{m}_{i}\right)
$$

for which $q_{i}, \vec{r}_{i}$ and $\vec{m}_{i}$ are the atomic partial charge, position vector and intra-atomic dipole moment of the $i^{t h}$ atom, respectively. Examples of such formulations are Hirshfeld's partition and the Quantum Theory of Atoms in Molecules (QTAIM). Our group has employed the latter in IR-intensity studies for more than a decade now, so we'll follow the terminology that appeared in most of our previous works, changing only when the former name is no longer a good choice for a given mathematical structure.

The derivation of (16) with respect to the $k^{\text {th }}$ normal coordinate leads to:

$$
\begin{aligned}
\left(\frac{\partial \vec{p}}{\partial Q_{k}}\right) & =\sum_{j=1}^{N} \sum_{i=1}^{N}\left(\frac{\partial \vec{p}_{i}}{\partial \vec{r}_{j}}\right) \cdot\left(\frac{\partial \vec{r}_{j}}{\partial Q_{k}}\right) \\
& =\sum_{j=1}^{N}\left[\sum_{i=1}^{N} \frac{\partial}{\partial \vec{r}_{j}}\left(q_{i} \vec{r}_{i}+\vec{m}_{i}\right)\right]\left(\frac{\partial \vec{r}_{j}}{\partial Q_{k}}\right) \\
& =\sum_{j=1}^{N} \sum_{i=1}^{N}\left[\left(\frac{\partial\left(q_{i} \cdot \vec{r}_{i}\right)}{\partial \vec{r}_{j}}\right)+\left(\frac{\partial \vec{m}_{i}}{\partial \vec{r}_{j}}\right)\right]\left(\frac{\partial \vec{r}_{j}}{\partial Q_{k}}\right)
\end{aligned}
$$

The first derivative within the brackets gives rise to two terms:

$$
\left(\frac{\partial \vec{p}}{\partial Q_{k}}\right)=\sum_{j=1}^{N} \sum_{i=1}^{N}\left[\left(q_{i} \frac{\partial \vec{r}_{i}}{\partial \vec{r}_{j}}\right)+\left(\vec{r}_{i} \frac{\partial q_{i}}{\partial \vec{r}_{j}}\right)+\left(\frac{\partial \vec{m}_{i}}{\partial \vec{r}_{j}}\right)\right]\left(\frac{\partial \vec{r}_{j}}{\partial Q_{k}}\right)
$$

The first of three terms inside the brackets concerns a derivative whose result is conditioned to the Kroenecker's delta, $\delta_{i j}$, meaning it is equal to one if $i=j$ and zero otherwise. Thus:

$$
\left(\frac{\partial \vec{p}}{\partial Q_{k}}\right)=\sum_{j=1}^{N} \sum_{i=1}^{N}\left[\left(q_{i} \delta_{i j}\right)+\left(\vec{r}_{i} \frac{\partial q_{i}}{\partial \vec{r}_{j}}\right)+\left(\frac{\partial \vec{m}_{i}}{\partial \vec{r}_{j}}\right)\right]\left(\frac{\partial \vec{r}_{j}}{\partial Q_{k}}\right)
$$

Inside the brackets we have three terms called Charge, Charge Transfer and Dipolar Polarization, respectively. Combined, they compose the CCTDP method for evaluation of infrared intensities [17]. When firstly proposed the model was called Charge-Charge Flux-Dipole Flux [18], following a long term tradition based on fluxes, like the also famous Equilibrium Charge-Charge Flux (ECCF) [19, 20] and Charge-Charge Flux-Overlap (CCFO) [21] and its modified version (CCFOM) [22] models. We now believe the word flux can lead to some misconceptions and thus aim to avoid it, preferring to use charge transfer and polarization instead.

The overall sum over $i$ and $j$ in Eq. (21) lead us to:

$$
\left(\frac{\partial \vec{p}}{\partial Q_{k}}\right)=\left(\frac{\partial \vec{p}}{\partial Q_{k}}\right)^{C}+\left(\frac{\partial \vec{p}}{\partial Q_{k}}\right)^{C T}+\left(\frac{\partial \vec{p}}{\partial Q_{k}}\right)^{D P}
$$

This equation is very familiar to us since it appeared in erlier works regarding this model around 15 years ago. It concerns the CCTDP partition of the total molecular dipole moment derivative. The novely is that we can now access this information in an atom-by-atom approach for both the AC's and the DC's. Thus, by 
substituing one of the derivatives within Eqs. (14) and (15) by Eq. (22), one can reach the following expansion:

$$
\begin{aligned}
A_{k} & =\sum_{i=1}^{N} A_{i, k}^{C}+A_{i, k}^{C T}+A_{i, k}^{D P} \\
& =\sum_{j=1}^{N} A_{j, k}^{C}+A_{j, k}^{C T}+A_{j, k}^{D P}
\end{aligned}
$$

All the described ways of partitioning the IR intensities are related to one another, and Figure 3 show these relations in a direct visual approach, supposing a triatomic molecule $(N=3)$. The cube on Figure $3-a)$ shows the total IR intensity of a given mode while Figure $3-b)-f$ ) show the several ways in which we performed partitions of this total intensity. Even though different, its made clear that all of them need to reflect the same total intensity if the individual terms are summed together. If the molecule have a different number of atoms, the $i$ and $j$ axis will be proportional but the schematic partitions will be the same wathever the molecular size.
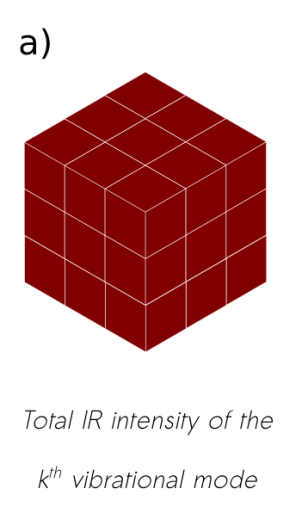

$k^{\text {th }}$ vibrational mode

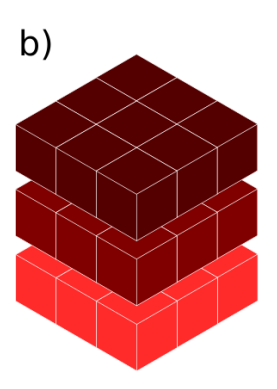

Molecular CCTDP

partition

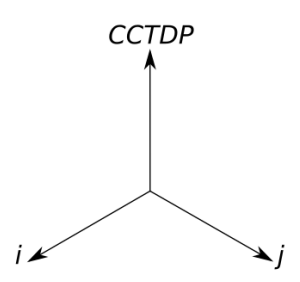

Figure 3: a) The cube represent the total intensity for the $k^{\text {th }}$ vibrational mode of a triatomic molecule, which will be partitioned in different but related ways: b) The molecular CCTDP partition of the IR intensity. c) The Atomic Contributions (AC's); d) The Dynamic Contributions (DC's); e) CCTDP partition of the AC's; f) CCTDP partition of the DC's.

\subsubsection{Charge-only models}

If the model used to obtain the atomic partial charges does not make use of atomic dipoles, all the terms in the previous equations regarding the derivatives of the atomic dipole will vanish, while the remaining ones kept inaltered. For instance, Eq. (22) would be just:

$$
\left(\frac{\partial \vec{p}}{\partial Q_{k}}\right)=\left(\frac{\partial \vec{p}}{\partial Q_{k}}\right)^{C}+\left(\frac{\partial \vec{p}}{\partial Q_{k}}\right)^{C T}
$$

The reader who are more familiar with these kind of analysis would readily recall the Equilibrium ChargeCharge Flux (ECCF) model since in Eq. (25) $C \equiv E C$ and $C T \equiv C F$. Consequently, the analogous, on this 
approach, to Eqs. (24) and (23) would be:

$$
\begin{aligned}
A_{k} & =\sum_{i=1}^{N} A_{i, k}^{C}+A_{i, k}^{C T} \\
& =\sum_{j=1}^{N} A_{j, k}^{C}+A_{j, k}^{C T}
\end{aligned}
$$

At first it would seem that Eqs. (26) and (27) would lead to a simpler analysis than Eqs. (23) and (24) due to fewer terms, but we stress the very opposite: atomic polarizations are necessary for coherent IR intensity interpretations since they furnish an additional (and absolutely necessary) degree of freedom to the description of the electronic density [23]. While CCT (of ECCF, as earlier named) models can reproduce the total intensity as a sum of terms, the interpretation obtained from these terms (the information therein) is quite often against what is expected from elementary chemical concepts. We could draw a scheme similar to Figure 3 for which the only difference would be two rather three terms (C and CT) in the vertical axis.

\section{Computational Details}

Handle all these equations by hand is by no means an easy task. Therefore, the entire protocol just presented was fully implemented on the Placzek program [24, 25]. This code already performed earlier versions of the molecular CCTDP decomposition, like Eqs. (21) and (22), but now the complete AC/DC analysis is also readily available. The user can access the total AC/DC contributions in $\mathrm{km}^{\mathrm{mol}} \mathrm{mol}^{-1}$ (Eqs. (14) and (15)) as well as their CCTDP terms (following Eqs. (23) and (24)). Placzek is made available free of charge and a couple of examples of the required inputs can be obtained by the address given in the Supplementary Information. Placzek works along with Gaussian [26] for the electronic structure calculations and with AIMAll [27] and Morphy98 [28] to integrate the electron densities to obtain the QTAIM charges and dipoles.

The following numerical results were obtained using the same approach already used in earlier works on the CCTDP model. The diference is now Placzek prints additional information, while the input protocol is somewhat the same. The geometry was optimized using Gaussian09, which also provided the raw infrared frequencies and intensities and the Hessian matrix. Starting from the optimized structure, Placzek generates a set of $6 N$ distorted geometries $( \pm 0.001 \AA$ at each Cartesian direction for each of the $N$ atoms), and each of these geometries had their QTAIM charges evaluated by AIMAll, by integration of the respective electron density from the wavefunction files. Placzek then run the numerical derivatives needed and print the complete analysis. The reader who prefer to handle the equations by him/herself must notice the $6 \mathrm{~N}$ geometries are required only to compute the Atomic Contributions; the Dynamic Contributions can be evaluated directly from the equilibrium geometry (assuming the Atomic Polar Tensors and normal coordinates in the Cartesian space are available).

\section{Examples of representative numerical results}

Given the enourmous amount of information given in the aforementioned sections, we present now the results, obtained with Placzek, for water. Although none of the above equations are restricted to a particular molecular size or shape, it is not our goal to explore various molecules and actually interpret the results here, but rather just present a concise computational protocol to achieve them.

Table 1 shows the AC/DC results for water, using three different partitions: QTAIM and Hirshfeld (with both atomic charges and atomic dipoles) and ChelpG (just atomic charges, without atomic dipoles). The level of theory used was QCISD/aug-cc-pVTZ. The default grids were used on AIMAll and Gaussian to obtain the atomic charges and dipoles.

The results in Table 1 furnish a numerical proof for some situations already anticipated from the equations. 
Table 1: Atomic (AC's) and Dynamic (DC's) Contributions (all values in $k m . \mathrm{mol}^{-1}$ ) for the vibrations of water at QCISD/aug-cc-pVTZ level using the following population analyses: QTAIM and Hirshfeld (employing both charges and dipoles) and ChelpG (only charges).

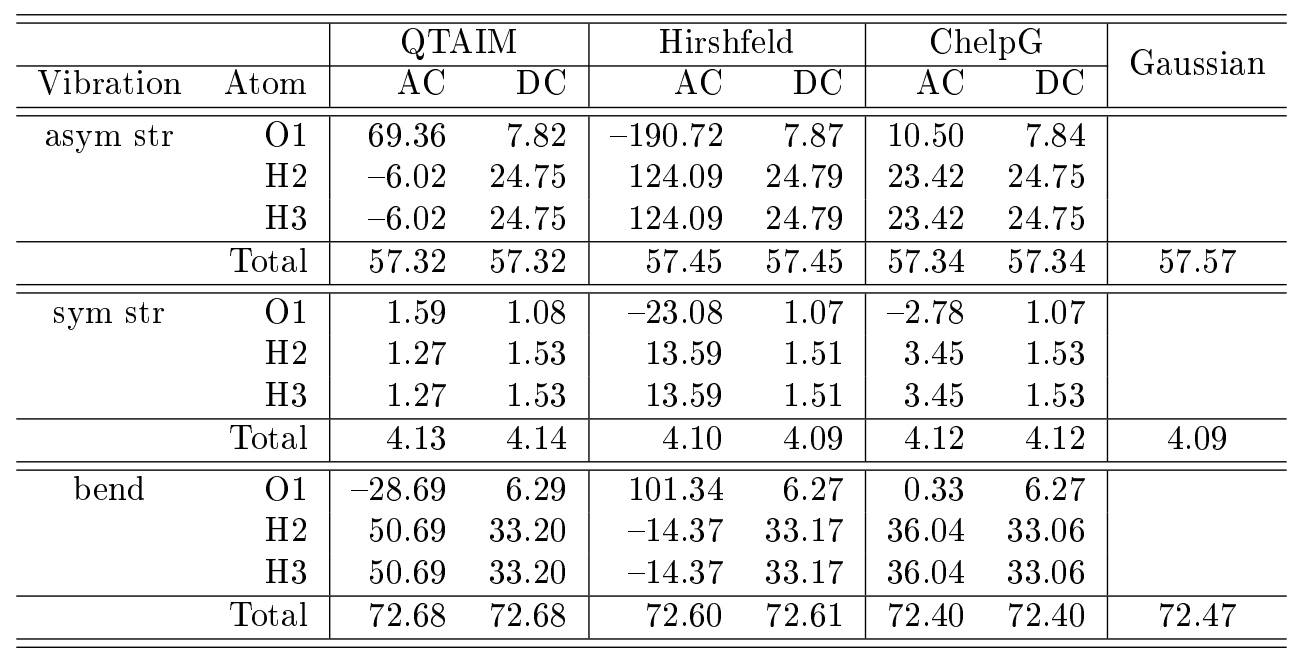

First of all, all sets of AC/DC's sum to the same total intensity (row Total), which is also the same value derivered directly by Gaussian (in the last column). The small differences between the values from AC/DC's and Gaussian's arise from the numerical procedures employed by the programs in obtaining not only the charges/dipoles but also when performing the required derivatives. Moreover, the differences are small enough to not harm the conceptual interpretations, which are our main goals. It is worth to mention that the results also respect the molecular symmetry, since symmetrically equivalent atoms show equal numerical results for their AC/DC's.

Another key aspect that Table 1 makes evident is that, for a given vibration, DC's are expected to be equal, regardless the charge model chosen, but the AC's are expected to be different for each charge model. One can see the DC's obtained using any of the three models employed (QTAIM, Hirshfeld and ChelpG) are essentialy equal, even though QTAIM and Hirshfeld make use of atomic dipoles and ChelpG does not. On the other hand, all sets of AC's are remarkably different from each other, as expected since the three models are based on very different theoretical approaches. This observation reinforces how critical is the selection of the charge model for theoretical works. Moreover, the differences are not related solely to the presence or not of atomic dipoles, since QTAIM and Hirshfeld are as different to each other as are QTAIM/ChelpG and Hirshfeld/ChelpG.

Considering the AC's from the different charge models are already far different from one another, we could expect at least the same for their individual CCTDP (or, in case of ChelpG, CCT only) contributions, following Eqs. (23) and (24) (and Eqs. (26) and (27) for charge-only model ChelpG). Table 2 show the AC's and DC's partitioned by means of QTAIM/CCTDP, Hirshfeld/CCTDP and ChelpG/CCT models for the asymmetric stretch of water.

As expected, the general profile is remarkably different among the three data sets. We can see, for example, that all three models show total DC terms that are essentially equal to each other, but their inner parts $\left(D C_{C}, D C_{C T}\right.$ and $D C_{D P}$ are all different. This is important because demonstrates how DC's are immune to the partition scheme chosen for the atomic charges and dipoles, but DC's partitioned terms are not. Similar differences among the models are reflected in the AC's splitted terms, reinforcing the crucial step of selecting the charge model. Since AC's and DC's can be viewed as splitting the molecular dipole moment derivative into terms owing the individual atoms, it is important to notice that the sum of $C, C T$ and $D P$ (sum in the columns) over the atoms is equal for AC's and DC's (i.e. the sum of all charge terms, $C$, from AC's and DC's under QTAIM partition is equal, $169.69 \mathrm{~km}_{\mathrm{mol}}^{-1}$ ), and the same is veryfied for $C T$ 's and $D P$ 's. This is very important because when summed over the atoms, both AC and DC sets need to sum to the same values, namely the molecular dipole moment derivative. These observations stress an internal coherence of the equations and the results obtained through them. AC/DC analysis is then a valuable tool for interpreting IR intensities at the atomic level, given all sort of interpretations that are made available through it. 
Table 2: Atomic (AC's) and Dynamic (DC's) Contributions for asymmetric stretch of water, partitioned using QTAIM/CCTDP, Hirshfeld/CCTDP and ChelpG/CCT models. All values are in $\left(\mathrm{km}_{\mathrm{m}} \mathrm{mol}^{-1}\right)$.

\begin{tabular}{|c|c|c|c|c|c|c|c|c|}
\hline QTAIM & \multicolumn{4}{|c|}{$\overline{\mathrm{AC}}$} & \multicolumn{4}{|c|}{$\overline{\mathrm{DC}}$} \\
\hline CCTDP & $\mathrm{C}$ & $\mathrm{CT}$ & DP & Total & $\mathrm{C}$ & $\mathrm{CT}$ & $\mathrm{DP}$ & Total \\
\hline O1 & 18.99 & 0.00 & 50.36 & 69.36 & 18.99 & -12.37 & 1.21 & 7.83 \\
\hline $\mathrm{H} 2$ & 75.35 & -84.80 & 3.43 & -6.02 & 75.35 & -78.61 & 28.01 & 24.75 \\
\hline H3 & 75.35 & -84.80 & 3.43 & -6.02 & 75.35 & -78.61 & 28.01 & 24.75 \\
\hline Total & 169.69 & -169.59 & 57.22 & 57.33 & 169.69 & -169.59 & 57.22 & 57.32 \\
\hline Hirshfeld & \multicolumn{4}{|c|}{$\mathrm{AC}$} & \multicolumn{4}{|c|}{$\mathrm{DC}$} \\
\hline CCTDP & $\mathrm{C}$ & $\mathrm{CT}$ & DP & Total & $\mathrm{C}$ & $\mathrm{CT}$ & DP & Total \\
\hline O1 & 4.72 & 0.00 & -195.44 & -190.72 & 4.72 & 3.74 & -0.60 & 7.87 \\
\hline $\mathrm{H} 2$ & 18.74 & 25.58 & 79.76 & 124.09 & 18.74 & 23.71 & -17.66 & 24.79 \\
\hline H3 & 18.74 & 25.58 & 79.76 & 124.09 & 18.74 & 23.71 & -17.66 & 24.79 \\
\hline Total & 42.20 & 51.16 & -35.92 & 57.45 & 42.20 & 51.16 & -35.92 & 57.45 \\
\hline ChelpG & \multicolumn{4}{|c|}{$\overline{\mathrm{AC}}$} & \multicolumn{4}{|c|}{$\overline{\overline{\mathrm{DC}}}$} \\
\hline $\mathrm{CCT}$ & $\mathrm{C}$ & $\mathrm{CT}$ & DP & Total & $\mathrm{C}$ & $\mathrm{CT}$ & $\mathrm{DP}$ & Total \\
\hline O1 & 10.50 & 0.00 & - & 10.50 & 10.50 & -2.66 & - & 7.84 \\
\hline $\mathrm{H} 2$ & 41.68 & -18.26 & - & 23.42 & 41.68 & -16.93 & - & 24.75 \\
\hline H3 & 41.68 & -18.26 & - & 23.42 & 41.68 & -16.93 & - & 24.75 \\
\hline Total & 93.86 & -36.52 & - & 57.34 & 93.86 & -36.52 & - & 57.34 \\
\hline
\end{tabular}

To finish, one call attention to how different can be the interpretations from different charge models: while the molecular $C$ and $C T$ terms within the QTAIM approach almost perfectly cancell each other (169.69 - 169.59 $=0.01 \mathrm{~km} \cdot \mathrm{mol}^{-1}$ ), making the total intensity solely described by the polarization term, $D P$, this polarization term does not exist within the ChelpG/CCT partition. So the most important term from a given approach may be actually absent in another. Thus, we advise maximum care on picking up the charge model.

\section{Conclusions}

We presented a broad and comprehensive analysis of infrared intensities at the atomic level. All terms are singleatom quantities which can be evaluated in the same units as the total intensities (in principle, $\mathrm{km}^{\mathrm{mol}} \mathrm{l}^{-1}$, but any other unit is also readily accessible). Moreover, the two different ways in which the total intensity can be splitted (AC or DC) will sum to overall equivalent results (the total intensity), while they are inherently different in terms of the kind of information they bring to us. AC's (total or splitted into smaller terms regarding the partition scheme selected for atomic charges or atomic charges and dipoles) will reflect the features of these charge models. On the other hand, the total DC's are not influenced by these models and can actually be defined directly from the total molecular dipole moment derivative, while the splitted DC's also behave following the chosen partition scheme.

Nonetheless, both AC's and DC's are invariant to translation/rotation and will be only affected by the electronic environment in which the atoms they belong are inserted. This analysis encloses two complementary ways of assessing information about electronic structure from electronic calculations of IR intensity, whose accuracy can be guided by the total IR intensities available experimentally. Even though our group is focused in interpreting IR intensities for more than 40 years now, to the best of our knowledge this is the first time such approach is presented, suggesting there is a whole new source of information to be pursued in this field.

To make easier the calculation of all these quantities to the less experienced reseacher, a completely automated computational protocol is presented, free of charge and with examples of input construction. We hope to get the attention and collaboration of our peers in exploiting the new possibilities opened with this analysis. 


\section{Acknowledgements}

LJD thanks Fundação de Amparo à Pesquisa do Estado de São Paulo (FAPESP) for a BEPE doctoral fellowship (2018/24844-7). REB acknowledges FAPESP for funding research grant (2018/08861-9) and Conselho Nacional de Desenvolvimento Científico e Tecnológico (CNPq) for a research fellowship (304518/2014-0). WER also thanks Fundação Araucária de Apoio ao Desenvolvimento Científico e Tecnológico (FAADCT/PR) for a research fellowship (89-2019/FA) and finantial support to computational facilities.

\section{References}

[1] A.F. Silva, W.E. Richter, A.B.M.S. Bassi, and R.E. Bruns. Physical Chemistry Chemical Physics, 17:30378-30388, 2015.

[2] E.B. Wilson Jr., J.C. Decius, and P.C. Cross. Molecular Vibrations: The Theory of Infrared and Raman Vibrational Spectra. Dover Books on Chemistry, 1955.

[3] B.S. Galabov and T. Dudev. Vibrational Spectra and Structure Vol 22: Vibrational Intensities. Elsevier Science, 1996.

[4] R.S. Mulliken. Journal of Chemical Physics, 23:1833, 1955.

[5] J.A. Montgomery, M.J. Frisch, J.W. Ochterski, and G.A. Petersson. Journal of Chemical Physics, 112:6532, 2000.

[6] U.C. Singh and P.A. Kollman. Journal of Computational Chemistry, 5:129, 1984.

[7] A.E. Reed, R.B. Weinstock, and F. Weinhold. Journal of Chemical Physics, 83:735, 1985.

[8] L.E. Chirlian and M.M. Francl. Journal of Computational Chemistry, 8:894, 1987.

[9] C.M. Breneman and K.B. Wiberg. Journal of Computational Chemistry, 11:361, 1990

[10] A.J. Stone and M. Alderton. Molecular Physics, 56:1047, 1985.

[11] J. Cioslowski. Journal of the American Chemical Society, 111:8333, 1989.

[12] C.F. Guerra, J.-W. Handgraaf, E.J. Baerends, and F.M. Bickelhaupt. Journal of Computational Chemistry, 25:189, 2004.

[13] A.V. Marenich, S.V. Jerome, C.J. Cramer, and D.G. Trhular. Journal of Chemical Theory and Computation, 8:527, 2012.

[14] F.L. Hirshfeld. Theoretica Chimica Acta, 44:129, 1977.

[15] P. Bultinck, C.V. Alsenoy, P.W. Ayers, and R. Carbo-Dorca. Journal of Chemical Physics, 126:144111, 2007.

[16] R.F.W. Bader. Atoms in Molecules: a quantum theory. Clarendon Press, Oxford, 1994.

[17] A.F. Silva, W.E. Richter, H.G.C. Meneses, and R.E. Bruns. Physical Chemistry Chemical Physics, 16:23224, 2014.

[18] R.L.A. Haiduke and R.E. Bruns. The Journal of Physical Chemistry A, 109:2680, 2005.

[19] J.C. Decius. Journal of Molecular Spectroscopy, 57:348, 1975.

[20] A. Milani and C. Castiglioni. Journal of Molecular Structure:THEOCHEM, 955:158, 2010.

[21] W.T. King and G.B. Mast. Journal Physical Chemistry, 88:2521, 1986.

[22] M. Gussoni, M.N. Ramos, C. Castiglioni, and G. Zerbi. Chemical Physics Letters, 142:515, 1987.

[23] W.E. Richter, A.F. Silva, and R.E. Bruns. Journal of Chemical Physics, 146:134107, 2017.

[24] T.C.F. Gomes, J.V. Silva, L.N. Vidal, P.A.M. Vazquez, and R.E. Bruns. Theoretical Chemistry Accounts, 121:173, 2008.

[25] T.C.F. Gomes, J.V. Silva, L.N. Vidal, P.M.A. Vazquez, and R.E. Bruns. Química Nova, 31:1750, 2008.

[26] M.J. Frisch, G.W. Trucks, H.B. Schlegel, G.E. Scuseria, M.A. Robb, J.R. Cheeseman, G. Scalmani, V. Barone, B. Mennucci, G.A. Petersson, H. Nakatsuji, M. Caricato, X. Li, H.P. Hratchian, A.F. Izmaylov, J. Bloino, G. Zheng, J.L. Sonnenberg, M. Hada, M. Ehara, K. Toyota, R. Fukuda, J. Hasegawa, M. Ishida, T. Nakajima, Y. Honda, O. Kitao, H. Nakai, T. Vreven, Jr. J.A. Montgomery, J.E. Peralta, F. Ogliaro, M. Bearpark, J.J. Heyd, E. Brothers, K.N. Kudin, V.N. Staroverov, R. Kobayashi, J. Normand, K. Raghavachari, A. Rendell, J.C. Burant, S.S. Iyengar, J. Tomasi, M. Cossi, N. Rega, J.M. Millam, M. Klene, J.E. Knox, J.B. Cross, V. Bakken, C. Adamo, J. Jaramillo, R. Gomperts, R.E. Stratmann, O. Yazyev, A.J. Austin, R. Cammi, C. Pomelli, J.W. Ochterski, R.L. Martin, K. Morokuma, V.G. Zakrzewski, G. A. Voth, P. Salvador, J.J. Dannenberg, S. Dapprich, A.D. Daniels, Ö. Farkas, J.B. Foresman, J.V. Ortiz, J. Cioslowski, and D.J. Fox. Gaussian09, Revision B.01. Gaussian, Inc., Wallingford CT, 2009.

[27] T.A. Keith. Aimall (version 14.11.23), tk gristmill software, overland park ks, usa, 2014 (aim.tkgristmill.com).

[28] P.L.A. Popelier. Morphy98, a program written by p.l.a. popelier with a contribution from r.g.a. bone. umist, manchester, england, eu., 1998.

\section{Supplementary Information}

Program PLACZEK is made available upon request by contacting the developer, Prof. Dr. Luciano N. Vidal (lnvidal@utfpr.edu.br). It's ensured the access to the main program and examples of inputs for the complete AC/DC analysis (CCTDP or only CCT) based on GAUssian (electronic structure calculations and ordinary atomic charge partitions, like ChelpG or Hirshfeld) and AIMAll for the QTAIM analyses. 